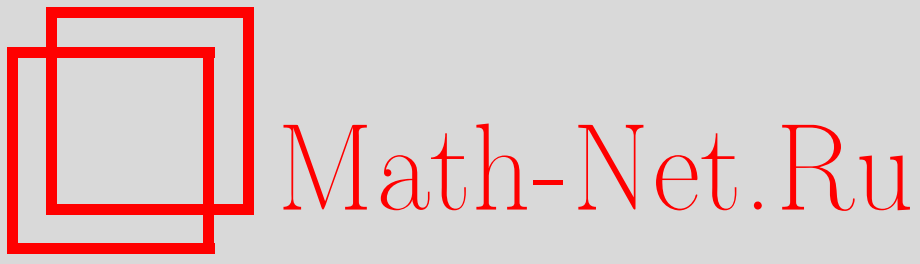

А. М. Вершик, Н. В. Цилевич, Марковские меры на таблицах Юнга и индуцированные представления бесконечной симметрической группы, Теория вероятн. и ее примен., 2006, том 51, выпуск 1, 4763

DOI: https://doi.org/10.4213/tvp145

Использование Общероссийского математического портала MathNet.Ru подразумевает, что вы прочитали и согласны с пользовательским соглашением

http://www . mathnet.ru/rus/agreement

Параметры загрузки:

IP : 52.87 .193 .239

26 апреля 2023 г., 14:22:22

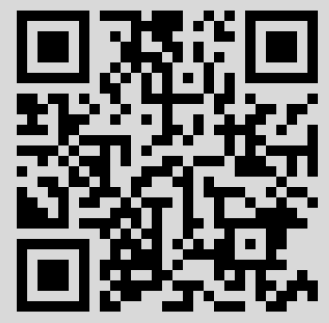




\title{
МАРКОВСКИЕ МЕРЫ НА ТАБЛИЦАХ ЮНГА И ИНДУЦИРОВАННЫЕ ПРЕДСТАВЛЕНИЯ БЕСКОНЕЧНОЙ СИММЕТРИЧЕСКОЙ ГРУППЫ ${ }^{1)}$
}

\begin{abstract}
В работе доказано, что класс так называемых марковских представлений бесконечной симметрической группы $\mathfrak{S}_{\mathbf{N}}$, ассоциированных с марковскими мерами на пространстве бесконечных таблиц Юнга, совпадает с классом простых представлений, т.е. индуктивных пределов представлений с простым спектром. Спектральная мера произвольного представления группы $\mathfrak{S}_{\mathbf{N}}$ с простым спектром эквивалентна мультимарковской мере на пространстве таблиц Юнга. Также показано, что представления группы $\mathfrak{S}_{\mathbf{N}}$, индуцированные с единичных представлений двучленных подгрупп Юнга, являются марковскими, и найдены явные формулы для переходных вероятностей соответствующей марковской меры. Для изучения индуцированньх представлений используется тензорная модель двустрочечных представлений симметрических групп; в частности, найдены явные формулы для базиса Гельфанда-Цетлина в тензорных моделях.
\end{abstract}

Ключевые слова и Фразы: марковские меры, таблицы Юнга, индуцированные представления, простой спектр.

1. Введение. Рассмотрим граф Юнга $\mathbb{Y}$, т.е. $\mathbf{Z}_{+}$-градуированный граф диаграмм Юнга: вершины его $n$-го этажа $\mathbb{Y}_{n}$ суть диаграммы Юнга с $n$ клетками, а дуги соединяют две вершины соседних этажей, если соответствуюшие диаграммы отличаются на одну клетку. Пространство $T$ таблиц Юнга, т.е. бесконечных путей в графе Юнга, есть вполне несвязный (нестационарный) марковский компакт, и марковские меры на нем определяются обычным образом. Иначе говоря, для любой фиксированной диаграммы $\lambda_{n} \in \mathbb{Y}_{n}$ условная мера на множестве таблиц, «проходяших» на $n$-м шаге через эту диаграмму, есть прямое произведение условных мер на «прошлом» (до момента $n$ ) и «будущем» (после момента $n$ ), т.е. прошлое и будушее пути при фиксированной «настоящей» диаграмме независимы. Такая мера определяется условными

* С.-Петербургское отделение Математического института им. В.А. Стеклова PAH; e-mail: vershik@pdmi.ras.ru, natalia@pdmi.ras.ru

1) Работа выполнена при поддержке грантов CRDF RUM1-2622-ST-04 и INTAS 03-51-5018. 
вероятностями перехода (или коперехода) от одной диаграммы к следующей. В свою очередь это означает, что последовательность случайных величин $X_{n}$ (где $X_{n}(t)$ есть содержание $n$-й клетки таблицы $t$ ) имеет специальную структуру: распределение величины $X_{n+1}$ зависит не от всех предыдуших значений $X_{k}$, а только от «граничных» (от диаграммы).

Хвостовое разбиение $\xi$ на пространстве путей $T$ есть разбиение на классы конфинальных путей. Мера $\mu$ (не обязательно марковская) на $T$ называется эргодической (относительно хвостового разбиения), если всякое измеримое $\xi$-множество имеет либо нулевую, либо полную $\mu$-меру. Мера $\mu$ называется квазиинвариантной, если она квазиинвариантна в обычном смысле слова относительно любых преобразований (замен) начала таблицы. Иначе говоря, для почти любой таблицы $t$ и любого $n \in \mathbf{N}$ условная мера на множестве таблиц, совпадающих с таблицей $t$ начиная с момента $n$, строго положительна.

Напомним (см. [1], [2], [8]), что бесконечная симметрическая группа $\mathfrak{S}_{\mathbf{N}}$ есть индуктивный предел конечных симметрических групп $\mathfrak{S}_{n} \mathrm{c}$ естественными вложениями; диаграммы Юнга с $n$ клетками параметризуют неприводимые комплексные представления группы $\mathfrak{S}_{n}$; граф Юнга есть граф ветвления неприводимых представлений симметрических групп (или диаграмма Браттели групповой алгебры группы $\mathfrak{S}_{\mathbf{N}}$ ). Известно (см. [8]), что всякая эргодическая квазиинвариантная мера на пространстве бесконечных таблиц $T$ и 1-коцикл (на хвостовом отношении эквивалентности в этом пространстве) со значениями в группе комплексных чисел, равных по модулю единице, определяют неприводимое представление бесконечной симметрической группы $\mathfrak{S}_{\mathbf{N}} \mathbf{c}$ простым спектром (относительно алгебры Гельфанда-Цетлина) по формуле (2) (см. п. 3). И наоборот, всякое неприводимое представление с простым спектром может быть реализовано в такой форме. При этом два таких представления эквивалентны тогда и только тогда, когда соответствуюшие им меры взаимно абсолютно непрерывны, а коциклы когомологичны. $\mathrm{He}$ все неприводимые представления получаются таким образом - существуют неприводимые представления, имеюшие непростой спектр; но указанные представления составляют естественный и важный класс неприводимых представлений, который определен инвариантным образом, т.е. замкнут относительно всех автоморфизмов группы $\mathfrak{S}_{\mathbf{N}}$.

Представление группы $\mathfrak{S}_{\mathbf{N}}$, определяемое марковской мерой, называется марковским представлением; тем самым, задать марковское представление можно, задав переходные вероятности добавления новой клетки к диаграмме Юнга (или копереходные вероятности удаления клетки).

Определим теперь обобщенно марковскую меру на пространстве $T$ как меру, для которой существует такая возрастающая последовательность $N_{1}, N_{2}, \ldots$ натуральных чисел, что при любом $n$ условные меры 
на прошлом и будущем независимы при фиксации таблищ на интервале $N_{n}, \ldots, N_{n+1}-1$.

По аналогии с соответствующей теоремой из работы [6] о квазиинвариантных мерах на пространствах последовательностей, с помощью теоремы о сходимости мартингалов можно доказать следующую теорему: всякая квазиинвариантная эргодическая мера взаимно абсолютно непрерывна с обобщенно марковской квазиинвариантной эргодической мерой. Тем самым, для анализа представлений с простым спектром достаточно рассматривать только квазиинвариантные обобщенно марковские меры.

Назовем простым представлением группы $\mathfrak{S}_{\mathrm{N}}$ индуктивный предел последовательности представлений конечных симметрических групп $\mathfrak{S}_{n}, n=1,2, \ldots$, для каждого из которых разложение на неприводимые не имеет кратностей (т.е. предел представлений с простыми спектрами). Простые представления имеют простой спектр, но не исчерпывают всех представлений с простым спектром. Определение класса простых представлений уже существенно зависит от аппроксимации группы $\mathfrak{S}_{\mathbf{N}}$ конечными группами (см. конец п. 3), но, поскольку в данной работе рассматривается только фиксированная стандартная аппроксимация, мы используем термин «простое представление», не оговаривая, о какой аппроксимации идет речь. Оказывается (теорема 1), что класс простых представлений совпадает с классом марковских представлений. Этот несложный, но важный факт связывает совершенно различные на первый взгляд понятия.

В этой статье мы рассматриваем лишь двустрочечные диаграммы Юнга, которые заполняют небольшую часть графа Юнга, а именно «половину» графа Паскаля (вершины параметризуются парами натуральных чисел $(n, k)$, где $n$ - номер этажа, а $k$ - номер вершины, причем $k \leqslant[n / 2])$. Путь в таком графе (таблице) есть траектория простого блуждания на полурешетке $\mathbf{Z}_{+}$с отражением в нуле. Все предыдушие определения (марковские меры, представления и т.д.) можно ограничить на этот случай.

Представления конечных симметрических групп, определяемые двустрочечными диаграммами, наиболее распространены в приложениях; они имеют удобную модель - так называемую тензорную реализацию, в которой пространство представления есть пространство конечномерных тензоров; при этом валентность тензоров не превышает половину размерности (см. [4]). Именно эта модель используется далее.

Основной результат (теорема 5) состоит в том, что представления бесконечной симметрической группы, индуцированные с двучленных подгрупп Юнга, являются простыми и, следовательно, марковскими, причем явно найденные марковские спектральные меры для этих представлений суть законы замечательных и естественных случайных блужданий на полурешетке. 
Вериик А. М., Цилевич Н. В.

Этот результат иллюстрирует тезис из работы [7] о современном плодотворном сближении алгебраических и вероятностных конструкций: понятие индуцированного представления является одним из основных в теории представлений, в то время как марковские меры и процессы занимают центральное место в теории вероятностей. В нашей конструкции оба понятия оказались удивительным образом сплетенными между собой.

Работа спланирована следуюшим образом. В п. 2 мы напоминаем понятия и вводим обозначения, связанные с графом Юнга, в том числе - ключевое для данной работы понятие марковской меры на пространстве таблиц Юнга. В п. 3 вводятся понятия марковского вектора и марковского представления и доказывается теорема о совпадении классов марковских и простых представлений. Пункт 4 посвящен описанию тензорной модели двустрочечных представлений конечных симметрических групп. В п. 5 найдены явные формулы для элементов базиса Гельфанда-Щетлина в тензорной модели. Наконец, в п. 6 мы показываем, что представления бесконечной симметрической группы, индуцированные с единичных представлений двучленных подгрупп Юнга вида $\mathfrak{S}_{\left\{i_{1}, i_{2}, \ldots\right\}} \times \mathfrak{S}_{\left\{j_{1}, j_{2}, \ldots\right\}}$, являются марковскими, и находим в явном виде соответствующие спектральные меры.

2. Граф Юнга и марковские меры на пространстве его путей. В этом пункте мы напоминаем необходимые понятия и обозначения, связанные с графом Юнга и теорией представлений симметрических групп (см., например, [1], [2], [9]), и, в частности, понятие марковской меры на пространстве путей графа Юнга.

Мы обозначаем через $\mathfrak{S}_{n}$ симметрическую группу степени $n$ и через $\mathbf{C}\left[\mathfrak{S}_{n}\right]$ - групповую алгебру группы $\mathfrak{S}_{n}$.

Неприводимые представления симметрической группы $\mathfrak{S}_{n}$ параметризуются множеством $\mathbb{Y}_{n}$ диаграмм Юнга с $n$ клетками. Обозначим через $\pi_{\lambda}$ неприводимое унитарное представление группы $\mathfrak{S}_{n}$, соответствуюшее диаграмме $\lambda \in \mathbb{Y}_{n}$, и через $\operatorname{dim} \lambda-$ размерность представления $\pi_{\lambda}$.

Ветвление неприводимых представлений симметрических групп описывается графом Юнга $\mathbb{Y}$. Множество вершин $\mathbf{Z}_{+}$-градуированного графа $\mathbb{Y}$ есть $\bigcup_{n} \mathbb{Y}_{n}$, и две вершины $\mu \in \mathbb{Y}_{n-1}$ и $\lambda \in \mathbb{Y}_{n}$ соединены ребром тогда и только тогда, когда $\mu \subset \lambda$. По определению, нулевой этаж $\mathbb{Y}_{0}$ состоит из пустой диаграммы $\varnothing$.

Обозначим через $T_{\lambda}$ множество (состоящее из $\operatorname{dim} \lambda$ элементов) таблиц Юнга формы $\lambda \in \mathbb{Y}_{n}$, т.е. множество путей в графе Юнга от $\varnothing$ до $\lambda$. Пусть $T_{n}=\bigcup_{\lambda \in Y_{n}} T_{\lambda}-$ множество таблиц Юнга с $n$ клетками.

В соответствии с правилом ветвления неприводимых представлений симметрических групп, пространство $V_{\lambda}$ неприводимого предста- 
вления $\pi_{\lambda}$ раскладывается в сумму одномерных подпространств, параметризованных таблицами $u \in T_{\lambda}$. Базис $\left\{h_{u}\right\}_{u \in T_{\lambda}}$, составленный из векторов этих подпространств, называется базисом Гельфанда-Цетлина. Он является собственным базисом для алгебры Гельфанда-Цетлина $\mathrm{GZ}_{n}$ подалгебры в $\mathbf{C}\left[\mathfrak{S}_{n}\right]$, порожденной центрами $Z\left[\mathfrak{S}_{1}\right], \ldots, Z\left[\mathfrak{S}_{n}\right]$.

Пусть теперь $\mathfrak{S}_{\mathbf{N}}=\bigcup_{n=1}^{\infty} \mathfrak{S}_{n}=\underset{\lim }{\longrightarrow} \mathfrak{S}_{n}-$ бесконечная симметрическая группа с фиксированной структурой индуктивного предела конечных групп.

Обозначим через $T=\lim _{\longleftarrow} T_{n}$ пространство бесконечных таблиц Юнга (проективный предел пространств $T_{n}$ относительно естественных проекций, отбрасывающих «хвост» пути). В топологии покоординатной сходимости $T$ есть вполне несвязный метризуемый компакт. Хвостовое отношение эквивалентности $\sim$ на $T$ определяется следуюшим образом: пути $s=\left(\mu_{1}, \mu_{2}, \ldots\right)$ и $t=\left(\lambda_{1}, \lambda_{2}, \ldots\right)$ эквивалентны, если они совпадают начиная с некоторого места (конфинальны), т.е. $\mu_{k}=\lambda_{k}$ для достаточно больших $k$. Обозначим через $[t]_{n} \in T_{n}$ начальный отрезок длины $n$ пути $t \in T$. Для данной конечной таблицы $u \in T_{n}$ обозначим через $C_{u}=\left\{t:[t]_{n}=u\right\}$ соответствуюшее цилиндрическое множество; для $\lambda \in \mathbb{Y}_{n}$ обозначим через $C_{\lambda}=\left\{t: t_{n}=\lambda\right\}=\bigcup_{u \in T_{\lambda}} C_{u}$ множество всех путей, проходящих через вершину $\lambda$.

Мера $M$ на пространстве $T$ называется иентральной, если для любых $n \in \mathrm{N}, \lambda \in \mathbb{Y}_{n}$ и любых таблиц $u, v \in T_{\lambda}$ формы $\lambda$ меры соответствующих цилиндрических множеств равны: $M\left(C_{u}\right)=M\left(C_{v}\right)$ (эквивалентным образом, мера $M$ центральна, если она инвариантна относительно любых преобразований начала таблицы). Нетрудно убедиться, что копереходные вероятности $M\left(C_{\lambda} \cap C_{\Lambda}\right) / M\left(C_{\Lambda}\right)$ центральной меры зависят только от пары диаграмм $\lambda \subset \Lambda$ (и не зависят от меры) и равны $\operatorname{dim} \lambda / \operatorname{dim} \Lambda$. Центральные меры играют очень важную роль в теории представлений симметрических групп²).

Мера $M$ на пространстве $T$ называется марковской, если при любом $n \in \mathbf{N}$ выполняется следующее условие: для любых диаграмм $\lambda \in \mathbb{Y}_{n}$ и $\Lambda \in \mathbb{Y}_{n+1}$ таких, что $\Lambda \subset \lambda$, и для любого пути $u \in T_{\lambda}$ события $C_{u}$ («прошлое») и $C_{\Lambda}$ («будущее») независимы при условии $C_{\lambda}$ («при фиксированном настоящем»). Иными словами, случайная таблица $t=\left(\lambda_{1}, \lambda_{2}, \ldots\right)$, рассматриваемая как последовательность случайных величин $\lambda_{n}$, где $\lambda_{n}$ принимает значения в множестве $\mathbb{Y}_{n}$ диаграмм Юнга с $n$ клетками, является цепью Маркова в обычном смысле слова. В терминах переходных вероятностей это означает, что переходная вероятность $M\left(C_{\Lambda} \cap C_{u}\right) / M\left(C_{u}\right)$ зависит только от формы $\lambda$ таблицы $u$, но не от самой таблицы. Заметим, что марковость «вперед» и «назад»

2) В теории динамических систем такие меры называются мерами максимальной энтропии. 
эквивалентны, поэтому условие марковости можно аналоги'пым образом сформулировать в терминах копереходных вероятностей.

Нетрудно убедиться, что любая центральная мера является марковской. Таким образом, центральные меры образуют класс марковских мер с фиксированными копереходными вероятностями, равными $\operatorname{dim} \lambda / \operatorname{dim} \Lambda$.

3. Марковские векторы и марковские представления. Рассмотрим циклическое представление $\pi$ группы $\mathfrak{S}_{n}$ в пространстве $V$, имеющее простой спектр (т.е. раскладывающееся в сумму попарно неэквивалентных неприводимых представлений), и циклический вектор $\xi$ единичной длины в этом представлении. Рассмотрим пару диаграмм $\mu \supset \lambda$ с числом клеток $n$ и $n-1$ соответственно и предположим, что представление $\pi$ содержит подпредставление, эквивалентное $\pi_{\mu}$ (для простоты будем обозначать его тем же символом). Спроектируем вектор $\xi$ на пространство $V_{\mu}$ подпредставления $\pi_{\mu}$ и обозначим полученный вектор через $\xi_{\mu}$, а затем спроектируем $\xi_{\mu}$ на пространство подпредставления $\pi_{\lambda}$ группы $\mathfrak{S}_{n-1}\left(\right.$ содержащееся в $\left.V_{\mu}\right)$ и обозначим полученный вектор через $\xi_{\mu, \lambda}$. Отношение квадратов норм

$$
\left(\frac{\left\|\xi_{\mu, \lambda}\right\|}{\left\|\xi_{\mu}\right\|}\right)^{2}
$$

назовем копереходной вероятностью пары $\mu, \lambda$.

Определим меру на пространстве всех таблиц $t$ с диаграммами, соответствующими представлениям, входяшим в представление $\pi$ группы $\mathfrak{S}_{n}$, следующим образом: вероятность таблицы (т.е. пути) равна произведению копереходных вероятностей вдоль всего пути. Нетрудно видеть, что это определение корректно и что полученная мера есть спектральная мера вектора $\xi$ относительно алгебры Гельфанда-Цетлина; при этом величины (1) суть копереходные вероятности этой спектральной меры в обычном смысле.

Уточним также, что в наших рассуждениях циклический вектор может быть умножен на скалярный множитель, по модулю равный единице, - это не меняет меру; любое другое изменение циклического вектора меняет меру.

Будем говорить, что циклический вектор $\xi$ марковский, если его спектральная мера относительно алгебры Гельфанда-Цетлина является марковской.

Таким образом, марковость циклического вектора означает, что для любого $k<n$ и любой диаграммы $\lambda \in \mathbb{Y}_{k}$ вероятности любой таблицы (пути) с этой диаграммой не зависят от того, как таблица продолжена до уровня $n$. С точки зрения представлений и циклических векторов 
последнее означает, что норма проекции циклического вектора на подпространство представления группы $\mathfrak{S}_{k}$, эквивалентного $\pi_{\lambda}$, не зависит от того, каким путем мы пришли к данному подпространству.

Воспользуемся теперь следуюшей простой леммой теории представлений.

Лемма 1. Пусть в конечномерном гильбертовом пространстве $H$ задано унитарное представление группы $G$, которое примарно, т.е. разлагается в прямую (не обязательно ортогональную) сумму

$$
H=H_{1} \oplus \cdots \oplus H_{n}
$$

эквивалентных неприводимых представлений, $и$ в каждом из них задано по ииклическому вектору $v_{i} \in H_{i}, i=1, \ldots, n$. Следуюшие два утверждения эквивалентны.

1. Для любьх $i, j$ существует такая изометрия $T_{i, j}: H_{i} \rightarrow H_{j}$, сплетаюшая соответствуюшие представления, что $T_{i, j} v_{i}=v_{j}$.

2. В ииклической оболочке вектора $v=\sum v_{i}$ представление неприводимо.

Применяя эту лемму к прямой сумме подпредставлений, эквивалентных $\pi_{\lambda}$, мы получаем, что нормы проекции циклического вектора на соответствуюшие подпространства совпадают тогда и только тогда, когда $\pi_{\lambda}$ входит в разложение представления группы $\mathfrak{S}_{k}$ в циклической оболочке $\mathfrak{S}_{k} \xi$ вектора $\xi$ с единичной кратностью. Поскольку, как показано выше, совпадение норм проекций в свою очередь эквивалентно марковости, доказана следующая лемма о характеризации марковских векторов.

Лемма 2. Пусть $\pi$ - унитарное представление группы $\mathfrak{S}_{n}$ с простылм спектром. Циклический вектор $\xi$ представления $\pi$ является марковским тогда и только тогда, когда при любом $k<n$ представление группь $\mathfrak{S}_{k}$ в ииклической оболочке $\mathfrak{S}_{k} \xi$ вектора $\boldsymbol{\xi}$ относительно $\mathfrak{S}_{k}$ uмеeт простой спектр.

Заметим, что описанная процедура построения спектральной меры не задает меру на диаграммах, так как вероятности одной и той же диаграммы в разных таблицах, вообще говоря, различны. Если же эти вероятности совпадают, то спектральная мера является центральной.

Будем теперь рассматривать представления бесконечной симметрической группы $\mathfrak{S}_{\mathbf{N}}$.

Если даны квазиинвариантная мера $\mu$ на пространстве таблиц Юнга $T$ и 1-коцикл $c$ на парах конфинальных путей со значениями в группе комплексных чисел, по модулю равных единице, то мы можем построить унитарное представление группы $\mathfrak{S}_{\mathbf{N}}$ в пространстве $L^{2}(T, \mu)$ следующим образом (см., например, [8]). Напомним, что при помощи 
преобразования Фурье групповая алгебра $\mathbf{C}\left[\mathfrak{S}_{\mathbf{N}}\right]$ бесконечной симметрической группы реализуется как скрешенное произведение, построенное по коммутативной алгебре функций на пространстве таблиц $T$ (алгебре Гельфанда-Цетлина) и хвостовому отношению эквивалентности. Искомое представление задается формулой

$$
L_{g} h(s)=\sum_{t \sim s} \sqrt{\frac{d \mu(s)}{d \mu(t)}} \widehat{g}(s, t) c(s, t) h(t), \quad h \in L^{2}(T, \mu),
$$

где $\widehat{g}-$ функция на парах конфинальных путей, соответствующая элементу $g \in \mathfrak{S}_{\mathbf{N}}$ (преобразование Фурье элемента $g$ ). Заметим, что коцикл тривиален на пространстве конечных таблиц.

О п ре д е лени е 1. Представление бесконечной симметрической группы $\mathfrak{S}_{\mathbf{N}}$ называется простылм, если оно есть индуктивный ${ }^{3)}$ предел представлений конечных симметрических групп $\mathfrak{S}_{n}$ с простым спектром.

3 а ме ч а н и я. 1. Строго говоря, правильно говорить о «простоте представления относительно аппроксимации группы $\mathfrak{S}_{\mathbf{N}}$ последовательностью групп $\mathfrak{S}_{n}, n=1,2, \ldots$, с обычными вложениями». При изменении аппроксимации меняется и класс простых представлений. Но поскольку в данной статье используется только стандартная аппроксимация, мы опускаем это уточнение (см. также замечание ниже об обобщенно марковских мерах).

2. Мы используем термин «простое представление» в гораздо более широком смысле по сравнению с работой [8], где простыми назывались индуктивные пределы неприводимых представлений; мы будем называть такие представления элементарными. Разумеется, элементарные представления являются простыми в нашем смысле.

Отметим, что представление с простым спектром является циклическим, т.е. содержит циклический вектор.

О п р е д е л е н и е 2. Представление с простым спектром бесконечной симметрической группы $\mathfrak{S}_{\mathbf{N}}$ называется марковским, если в пространстве представления существует циклический вектор, спектральная мера которого (относительно алгебры Гельфанда-Цетлина) является марковской.

Заметим, что представление с простым спектром является марковским тогда и только тогда, когда в его реализации (2) мера $\mu-$ марковская.

3) Напомним, что под индуктивным пределом унитарных представлений индуктивной цепочки конечных групп $G_{1} \subset G_{2} \subset \cdots$ понимается представление групшы $G=\bigcup G_{k}$ в гильбертовом пространстве, являющемся пополнением цепочки пространств представлений групп $G_{k}$ с (эквивариантными) изометрическими вложениями. 
Теорема 1. Представление бесконечной симметрической группы является марковским тогда и только тогда, когда оно простое.

Д о к а з а т е л ь с т в о. Если $\xi$ - марковский циклический вектор, то при любом $n$ представление группы $\mathfrak{S}_{n}$ в циклической оболочке $\mathfrak{S}_{n} \xi$ - марковское, а значит, по лемме 2 , имеет простой спектр. Таким образом, рассматриваемое представление есть предел представлений с простым спектром. Обратно, если имеется индуктивный предел представлений с простым спектром, то по лемме 2 вектор, полученный последовательными вложениями из единичного вектора в исходном одномерном представлении группы $\mathfrak{S}_{1}$, является марковским циклическим вектором. Теорема 1 доказана.

Рассмотрим марковское представление $\pi$ и марковский циклический вектор $\xi$. Спектр представления группы $\mathfrak{S}_{n}$ в его циклической оболочке $\mathfrak{S}_{n} \xi$ прост (по лемме 2), и мы получаем аппроксимацию $\pi$ представлениями с простым спектром, но действие группы $\mathfrak{S}_{n}$ в этом представлении может отличаться от стандартной формы Юнга множителем, равным по модулю единице. Разумеется, подправив базис на такой множитель (введя «фазу»), мы получим стандартное действие, но может случиться, что сходящейся системы множителей нет и, следовательно, в записи предельного действия появится коцикл $c$ - как в формуле (2). Вопрос о сходимости фаз и есть вопрос о когомологичности коцикла единичному. Хорошо известно, что существует много коциклов, не когомологичных единичному. Таким образом, для заданной марковской меры есть много неэквивалентных представлений, отличающихся коциклом. Коцикл «измеряет отклонение» реализации представления от его стандартной реализации. Мы не останавливаемся здесь на этих интересных вопросах, так как во всех рассматриваемьгх далее реализациях представлений (тензорная модель, индуцированное представление) коцикл единичный.

Нетрудно понять, как перенести теорему 1 на случай обобщенно марковских мер. Предположим, что спектральная мера представления является обобщенно марковской с интервалами марковости $N_{1}, N_{2}, \ldots$ (т.е. для любого $k$ при фиксации таблиц с $N_{k-1}, \ldots, N_{k}-1$ клетками диаграммы, предшествуюшие моменту $N_{k-1}$ и следуюшие за моментом $N_{k}-1$, независимы); тогда соответствующее представление является простым, но уже относительно разреженной цепочки подгрупп $\mathfrak{S}_{1}, \mathfrak{S}_{N_{1}}, \mathfrak{S}_{N_{2}}, \ldots$. Иначе говоря, представление есть предел представлений с простым спектром последовательности групп $\mathfrak{S}_{N_{k}}, k \rightarrow \infty$. Как уже упоминалось, любая квазиинвариантная мера на пространстве таблиц эквивалентна обобщенно марковской мере, - доказательство этого факта дословно совпадает с доказательством аналогичного утверждения для квазиинвариантных мер в пространстве односторонних последовательностей (см. [6]). Поэтому всякое неприводимое представление 
группы $\mathfrak{S}_{\mathbf{N}}$ с простым спектром (относительно алгебры ГельфандаЦетлина) есть предел конечномерных представлений с простьлм спектром некоторой последовательности групп $\mathfrak{S}_{N_{k}}, k=1,2, \ldots$

4. Тензорная модель двустрочечных представлений. В этом пункте мы описываем так называемую тензорную модель двустрочечных представлений симметрической группы, которая была предложена первым автором и изучена в [4] (см. также [5]).

Пусть $0 \leqslant k \leqslant n$; обозначим через $F_{n, k}$ множество $k$-элементных подмножеств в $\{1, \ldots, n\}$. Если $I=\left\{i_{1}, \ldots, i_{k}\right\} \in F_{n, k}$, положим $x_{I}=$ $x_{i_{1}} \cdots x_{i_{k}}$.

Обозначим через $A_{n, k}=\left\{\sum_{I \in F_{n, k}} c_{I} x_{I}\right\}$ векторное пространство бесквадратных однородных форм степени $k$ от $n$ переменных. Это пространство можно отождествить с пространством симметрических тензоров валентности $k$ с нулевыми диагональными элементами над $n$ мерным пространством (форма $f=\sum_{I \in F_{n, k}} c_{I} x_{I}$ отождествляется с тензором $\left\{T_{j_{1}, \ldots, j_{k}}\right\}_{j_{1}, \ldots, j_{k}=1}^{n}$, где $T_{j_{1}, \ldots, j_{k}}=c_{\left\{j_{1}, \ldots, j_{k}\right\}}$, если индексы $j_{1}, \ldots, j_{k}$ попарно различны, и $T_{j_{1}, \ldots, j_{k}}=0$ в противном случае).

Обозначим через $\|\cdot\|$ норму, порожденную стандартным скалярным произведением в пространстве форм (тензоров), задаваемую формулой

$$
\|f\|^{2}=\sum_{I \in F_{n, k}}\left|c_{I}\right|^{2}, \quad f=\sum_{I \in F_{n, k}} c_{I} x_{I} \in A_{n, k} .
$$

Пусть $A_{n, k}^{0}$ - подпространство в $A_{n, k}$, задаваемое следуюшим обра3ом:

$$
A_{n, k}^{0}=\left\{\sum c_{I} x_{I} \in A_{n, k} \mid \sum_{j \notin J} c_{J \cup j}=0 \text { для любого } J \in F_{n, k-1}\right\} .
$$

Симметрическая группа $\mathfrak{S}_{n}$ действует на пространстве $A_{n, k}$ подстановками индексов:

$$
\sigma \cdot \sum_{I \in F_{n, k}} c_{I} x_{I}=\sum_{I \in F_{n, k}} c_{I} x_{\sigma I}, \quad \text { где } \sigma\left\{i_{1}, \ldots, i_{k}\right\}=\left\{\sigma\left(i_{1}\right), \ldots, \sigma\left(i_{k}\right)\right\},
$$

или, в тензорной форме, $\sigma\left\{T_{j_{1}, \ldots, j_{k}}\right\}=\left\{T_{j_{1}, \ldots, j_{k}}^{\prime}\right\}$, где $T_{j_{1}, \ldots, j_{k}}^{\prime}=$ $T_{\sigma^{-1}\left(j_{1}\right), \ldots, \sigma^{-1}\left(j_{k}\right)}$. Легко видеть, что подпространство $A_{n, k}^{0}$ инвариантно относительно этого действия. Заметим, что пространства $A_{n, k}^{0}$ и $A_{n, n-k}^{0}$ (а также $A_{n, k}$ и $A_{n, n-k}$ ) очевидным образом изометричны, а соответствуюшие представления группы $\mathfrak{S}_{n}$ эквивалентны.

Реализации представлений симметрических групп в пространствах тензоров (форм) будем называть тензорными реализачиями. Следуюшая теорема представляет собой известный факт, который в терминах удобной для нас тензорной модели был доказан в [4]. 
Теорема 2. Пусть $k \leqslant n / 2$.

(1) Представление симметрической группь $\mathfrak{S}_{n}$ в простран-

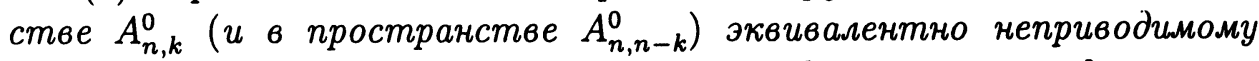
представлению $\pi_{n-k, k}$, соответствуюшему двустрочечной диаграмме $\lambda_{n, k}=(n-k, k)$ со строками длины $n-k u k$.

(2) Представление симметрической группь $\mathfrak{S}_{n}$ в пространстве $A_{n, k}$ эквивалентно прямой сумме (без кратностей) неприводимых представлений $\pi_{n-l, l}$ по всем $l=0,1, \ldots, k$. В иастности, представление группь $\mathfrak{S}_{n}$ в пространстве $A_{n,[n / 2]}$ эквивалентно прямой суме

$$
\bigoplus_{k=0}^{[n / 2]} \pi_{n-k, k}
$$

всех двустрочечных неприводимых представлений.

5. Базис Гельфанда-Цетлина в тензорных реализациях. В этом пункте мы выводим явные формулы для элементов базиса Гельфанда-Цетлина в тензорных реализациях двустрочечных представлений симметрических групп.

Заметим прежде всего, что подпространство $A_{n, k}^{0}$ может быть определено следуюшим образом.

Лемма 3. Пространство $A_{n, k}^{0}$ есть подпространство в $A_{n, k}$, состоящее из форм, инвариантных относительно одновременного сдвига переменных на константу. Оно натянуто на формы вида $\left(x_{i_{1}}\right.$ $\left.x_{j_{1}}\right) \cdots\left(x_{i_{k}}-x_{j_{k}}\right)$, где все индексы $i_{1}, \ldots, i_{k}, j_{1}, \ldots, j_{k}$ попарно различны.

Разумеется, формы вида $\left(x_{i_{1}}-x_{j_{1}}\right) \cdots\left(x_{i_{k}}-x_{j_{k}}\right)$ линейно зависимы, так что они образуют в $A_{n, k}^{0}$ переполненную систему. Мы будем называть их псевдомономами.

Заметим, что двустрочечная таблица Юнга $u$ однозначно определяется последовательностью $p_{1}<\cdots<p_{k}$ элементов ее второй строки.

Теорема 3. Рассмотрим двустрочечную таблииу Юнга $u \in T_{n}$, и пусть $p_{1}<\cdots<p_{k}$ - элементы ее второй строки. Тогда элемент $h_{u}^{0}$ базиса Гельфанда-Цетлина в пространстве $A_{n, k}^{0}$, отвечаюиий табличе и, задается формулой

$$
h_{u}^{0}=c_{u}^{0} \sum_{i_{1}, \ldots, i_{k}}\left(x_{i_{1}}-x_{p_{1}}\right) \cdots\left(x_{i_{k}}-x_{p_{k}}\right),
$$

где $c_{u}^{0}$ - нормировочная константа и сумма берется по всем индексам $i_{1}, \ldots, i_{k}$ от 1 до $n$ таким, что $i_{j}<p_{j}$ при всех $j=1, \ldots, k$ и все элементы $i_{1}, \ldots, i_{k}, p_{1}, \ldots, p_{k}$ попарно различны.

Д о к а 3 а т е л с т в о. Достаточно проверить, что при любом $l=1, \ldots, n$ форма $h_{u}^{0}$ является собственным вектором для действия 
$\mathrm{YJM}$-элемента $^{4)} X_{l}$ с собственным значением, равным $c_{l}(u)$, содержанию клетки таблицы $u$, содержащей элемент $l$. Это можно сделать при помоши прямых комбинаторных вычислений.

Можно доказать, что нормировочная константа в формуле (4) равна

$$
c_{u}^{0}=\frac{1}{\left(\prod_{j=1}^{k}\left(p_{j}-2 j+1\right)\left(p_{j}-2 j+2\right)\right)^{1 / 2}} .
$$

П р и м е $\mathrm{p} 1$. Рассмотрим таблицу $u$ формы $\lambda=\lambda_{n-k, k}$, у которой вторая строка содержит элементы $2,4, \ldots, 2 k$. Тогда

$$
h_{u}^{0}=c_{u}^{0} \cdot\left(x_{1}-x_{2}\right)\left(x_{3}-x_{4}\right) \cdots\left(x_{2 k-1}-x_{2 k}\right), \quad c_{u}^{0}=\frac{1}{2^{k / 2}} .
$$

Это единственная таблица формы $\lambda$, для которой соответствуюший элемент базиса Гельфанда-Цетлина $h_{u}^{0}$ является псевдомономом; мы будем называть ее хорошей таблицей формы $\lambda$. Для всех других таблиц той же формы $h_{u}^{0}$ является линейной комбинацией псевдомономов.

Обозначим через $H_{n, m}^{k}$ подпространство в $A_{n, m}$, в котором реализуется представление $\pi_{n-k, k}$. Таким образом,

$$
A_{n, m}=\bigoplus_{k=0}^{m} H_{n, m}^{k} .
$$

Обозначим через $\psi_{n}^{l}$ линейный оператор, который действует на мономы следуюшим образом:

$$
\psi_{n}^{l} x_{I}=x_{I} \sum_{\substack{j_{1}, \ldots, j_{1} \notin I \\ \text { различны }}} x_{j_{1}} \cdots x_{j_{l}} .
$$

Как следует из результатов работы [4],

$$
H_{n, m}^{k}=\psi_{n}^{m-k} A_{n, k}^{0},
$$

и $\psi_{n}^{m-k}$ есть изоморфизм пространств $H_{n, m}^{k}$ и $A_{n, k}^{0}$, сплетаюший соответствуюшие представления группы $\mathfrak{S}_{n}$.

Используя соотношение (7), из леммы 3 и теоремы 3 можно получить следуюшие утверждения.

Лемма 4. Пространство $H_{n, m}^{k}$ натянуто на формь вида $\left(x_{i_{1}}-\right.$ $\left.x_{j_{1}}\right) \cdots\left(x_{i_{k}}-x_{j_{k}}\right) x_{s_{1}} \cdots x_{s_{m-k}}$, где все индексы $i_{1}, \ldots, i_{k}, j_{1}, \ldots, j_{k}, s_{1}, \ldots$, $s_{m-k}$ попарно различны.

4) Напомним, что $l$-й элемент Юнга-Юциса-Мэрфи (ҮЈМ-элемент) задается формулой $X_{l}=(1, l)+(2, l)+\cdots+(l-1, l) \in \mathbf{C}\left[\mathfrak{S}_{n}\right]$, где, как обычно, $(i, j)$ обозначает транспозицию элементов $i$ и $j$. ҮЈМ-элементы $X_{l}, l=1, \ldots, n$, образуют мультипликативный базис алгебры Гельфанда-Цетлина $\mathrm{GZ}_{n}$. Относительно алгебр ГельфандаЦетлина, ҮЈМ-элементов и их роли в теории представлений симметрических групा cM. [10]. 
Теорема 4. Рассмотрим дөустрочечную таблииу Юнга $u \in T_{n}$, u пусть $p_{1}<\cdots<p_{k}$ - элементы ее второй строки. Тогда элемент $h_{u}$ базиса Гельфанда-Цетлина в пространстве $H_{n, m}^{k}$, отвечаюиий табличе $и$, задается формулой

$$
h_{u}=c_{u} \sum\left(x_{i_{1}}-x_{p_{1}}\right) \cdots\left(x_{i_{k}}-x_{p_{k}}\right) x_{s_{1}} \cdots x_{s_{m-k}},
$$

где $c_{u}$ - нормировочная константа, а сумма берется по всем индексам $i_{1}, \ldots, i_{k}, s_{1}, \ldots, s_{m-k}$ om 1 до $n$ таким, ито $i_{j}<p_{j}$ для всех $j=1, \ldots, k$ и все элементы $i_{1}, \ldots, i_{k}, p_{1}, \ldots, p_{k}, s_{1}, \ldots, s_{m-k}$ попарно различны.

П р и м е $\mathrm{p} 2$. Для хорошей таблицы $u$ формы $\lambda=\lambda_{n-k, k}$, рассмотренной в примере 1 , имеем

$$
\begin{gathered}
h_{u}=c_{u} \cdot\left(x_{1}-x_{2}\right)\left(x_{3}-x_{4}\right) \cdots\left(x_{2 k-1}-x_{2 k}\right) \sum_{\substack{s_{1}, \ldots, s_{m-k} \in\{2 k+1, \ldots, n\} \\
\text { pasличны }}} x_{s_{1}} \cdots x_{s_{m-k}}, \\
c_{u}^{2}=\frac{1}{2^{k} C_{n-2 k}^{m-k}} .
\end{gathered}
$$

6. Индуцированные представления. В этом пункте мы рассматриваем класс неприводимых представлений бесконечной симметрической группы $\mathfrak{S}_{\mathbf{N}}$, индуцированных с двучленных подгрупп Юнга. А именно, рассмотрим разбиение $\mathbf{N}=A \cup B$ натурального ряда на два подмножества и представление группы $\mathfrak{S}_{\mathbf{N}}$, индуцированное с единичного представления подгруппы Юнга $\mathfrak{S}_{A} \times \mathfrak{S}_{B}$. Нетрудно показать, что это представление неприводимо. Его можно описать в терминах тензорной модели следуюшим образом.

Разбиение $\mathbf{N}=A \cup B$ натурального ряда на два подмножества однозначно определяется бесконечной последовательностью $\xi=\xi_{1} \xi_{2} \cdots$ нулей и единиц («бесконечным тензором»), где $\xi_{i}=1$ при $i \in A$ и $\xi_{i}=0$ при $i \in B$. При этом рассматриваемое индуцированное представление эквивалентно естественному подстановочному представлению группы $\mathfrak{S}_{\mathbf{N}}$ на бесконечных последовательностях в циклической оболочке последовательности $\xi$, которое мы будем обозначать через $\pi_{\xi}$. Заметим, что орбита последовательности $\xi$ есть дискретное множество $O_{\xi}$ бесконечных последовательностей нулей и единиц, совпадаюших с $\xi$ начиная с некоторого места, и $\pi_{\xi}$ есть унитарное представление группы $\mathfrak{S}_{\mathbf{N}}$ в пространстве $l^{2}\left(O_{\xi}\right)$.

Для простоты будем предполагать, что число единиц среди первых $n$ элементов последовательности $\xi$ не превосходит $n / 2$. Нетрудно убедиться, что произвольный случай может быть сведен к этому, но мы опускаем соответствуюшие технические подробности.

Рассмотрим циклическую оболочку $\mathfrak{S}_{n} \xi$ последовательности $\xi$ относительно конечной симметрической группы $\mathfrak{S}_{n}$. Она естественно отождествляется с пространством $A_{n, m}$, где $m=m(n)$ есть число единиц 
среди первых $n$ элементов последовательности $\xi$. При этом скалярное произведение, индуцированное из $l^{2}\left(O_{\xi}\right)$, совпадает со стандартным скалярным произведением в $A_{n, m}$. Таким образом, представление $\pi_{\xi}^{n}$ группы $\mathfrak{S}_{n}$ в $\mathfrak{S}_{n} \xi$ унитарно эквивалентно тензорному представлению в пространстве $A_{n, m}$, причем элемент $\xi$ можно отождествить с мономом $x_{i_{1}} \cdots x_{i_{m}} \in A_{n, m}$, где $i_{1}, \ldots, i_{m}$ - номера позиций от 1 до $n$, на которых в последовательности $\xi$ стоят единицы. При этом имеется естественное вложение $\iota_{n}: \pi_{\xi}^{n} \hookrightarrow \pi_{\xi}^{n+1}$ : если $\xi_{n+1}=0$, то $\iota_{n}$ есть тождественное вложение $A_{n, m} \hookrightarrow A_{n+1, m}$; если $\xi_{n+1}=1$, то $\iota_{n}: A_{n, m} \rightarrow A_{n+1, m+1}$ есть умножение на $x_{n+1}$, т.е. $\iota_{n} f=x_{n+1} f$. Следующая лемма очевидна.

Лемма 5. Индуцированное представление $\pi_{\xi}$ группы $\mathfrak{S}_{\mathrm{N}}$ есть индуктивный предел тензорных представлений $\pi_{\xi}^{n}$ әрупn $\mathfrak{S}_{n}$.

Основным результатом этого пункта является следующая теорема.

Теорема 5. Спектральная мера $\mu_{\xi}$ ииклического вектора $\xi$ в представлении $\pi_{\xi}$ относительно алгебры Гельфанда-Цетлина есть марковская мера на пространстве Т бесконечных таблич Юнга, и ее переходные вероятности задаются следующей формулой. Обозначим через $m(n)$ число единии среди первых $n$ элементов последовательности $\xi$.

Ecлu $\xi_{n+1}=0$, mo

$$
\begin{aligned}
\operatorname{Prob}\left(\lambda_{n, k}, \lambda_{n+1, k}\right) & =\frac{n-m(n)-k+1}{n-2 k+1}, \\
\operatorname{Prob}\left(\lambda_{n, k}, \lambda_{n+1, k+1}\right) & =\frac{m(n)-k}{n-2 k+1} .
\end{aligned}
$$

$\operatorname{Ecsu} \xi_{n+1}=1$, mo

$$
\begin{aligned}
\operatorname{Prob}\left(\lambda_{n, k}, \lambda_{n+1, k}\right) & =\frac{m(n)-k+1}{n-2 k+1}, \\
\operatorname{Prob}\left(\lambda_{n, k}, \lambda_{n+1, k+1}\right) & =\frac{n-m(n)-k}{n-2 k+1} .
\end{aligned}
$$

Д о к а з а т е л ь с т в о. Как упомянуто выше, при любом $n$ представление $\pi_{\xi}^{n}$ реализуется в пространстве $A_{n, m}$, где $m=m(n)$ есть число единиц среди первых $n$ элементов последовательности $\xi$. В частности, при любом $l \leqslant n$ представление группы $\mathfrak{S}_{l}$ в соответствуюшей циклической оболочке $\mathfrak{S}_{l} \xi$ последовательности $\xi$ имеет простой спектр по теореме 2. Отсюда по лемме 2 следует, что $\mu_{\xi}$ - марковская мера.

Найдем явные формулы для переходных вероятностей меры $\mu_{\xi}$. Как следует из правила ветвления неприводимых представлений симметрических групп, для любой формы $f \in H_{n, m}^{k}$ имеем $\iota_{n} f=f_{n+1, k}+f_{n+1, k+1}$, где $f_{n+1, k} \in H_{n+1, m^{\prime}}^{k}, f_{n+1, k+1} \in H_{n+1, m^{\prime}}^{k+1}$ и $m^{\prime}=m(n+1)=m+\xi_{n+1}$.

Лемма 6. Пусть $f \in H_{n, m}^{k}$. Согласно (7), $f=\psi_{n}^{m-k} f_{0}$, где $f_{0} \in A_{n, k}^{0}$. Тогдa 
(a) eсли $\xi_{n+1}=0, m o$

$$
\begin{aligned}
f_{n+1, k} & =\frac{n-m-k+1}{n-2 k+1}\left(f+x_{n+1} \psi_{n}^{m-k-1} f_{0}\right), \\
f_{n+1, k+1} & =\frac{1}{n-2 k+1}\left((m-k) f-(n-m-k+1) x_{n+1} \psi_{n}^{m-k-1} f_{0}\right) \\
\text { (b) ecлu } & \xi_{n+1}=1, m o \\
f_{n+1, k} & =\frac{m-k+1}{n-2 k+1}\left(x_{n+1} f+\psi_{n}^{m-k+1} f_{0}\right), \\
f_{n+1, k+1} & =\frac{1}{n-2 k+1}\left((n-m-k) x_{n+1} f-(m-k+1) \psi_{n}^{m-k+1} f_{0}\right) .
\end{aligned}
$$

Д о к а з а т е л с с в о. Нетрудно проверить, что формы $f_{n+1, k}$ и $f_{n+1, k+1}$ лежат в нужных пространствах. Очевидно, их сумма равна $\iota_{n} f$.

Следствие 1. Пусть $f \in H_{n, m}^{k}$. Тогда

(a) ecлu $\xi_{n+1}=0$, mo

$$
\left\|f_{n+1, k}\right\|^{2}=\frac{n-m-k+1}{n-2 k+1}\|f\|^{2}, \quad\left\|f_{n+1, k+1}\right\|^{2}=\frac{m-k}{n-2 k+1}\|f\|^{2} ;
$$

(b) ecлu $\xi_{n+1}=1, m o$

$$
\left\|f_{n+1, k}\right\|^{2}=\frac{m-k+1}{n-2 k+1}\|f\|^{2}, \quad\left\|f_{n+1, k+1}\right\|^{2}=\frac{n-m-k}{n-2 k+1}\|f\|^{2} .
$$

Д о к а з а т е л в с т в о. Искомые формулы получаются из леммы 6 прямыми вычислениями с учетом очевидных соотношений $\left(f_{n+1, k}, f_{n+1, k+1}\right)=0 ;\left\|f_{n+1, k}\right\|^{2}+\left\|f_{n+1, k+1}\right\|^{2}=\|f\|^{2} ;\left(f, x_{n+1} \psi_{n}^{m-k-1} f_{0}\right)=0$ в случае $\xi_{n+1}=0$ и $\left(x_{n+1} f, \psi_{n}^{m-k+1} f_{0}\right)=0$ в случае $\xi_{n+1}=1$ (первые два соотношения выполнены по определению, а последнее - в силу того, что один из векторов состоит из мономов, содержащих $x_{n+1}$, а другой - из мономов, не содержащих $\left.x_{n+1}\right)$. Следствие 1 доказано.

Как следует из доказательства леммы $2, \operatorname{Prob}(\mu \mid \lambda)=\left(\left\|f_{\lambda}\right\| /\left\|f_{\mu}\right\|\right)^{2}$, поэтому формулы (10), (11) вытекают из следствия 1. Теорема 5 доказана.

Заметим, что все спектральные меры индуцированных представлений, рассматриваемые в теореме 5 , не являются центральными (кроме тривиального случая, когда одно из множеств разбиения пусто).

Следствие 2. Оператор $\psi_{n}^{m-k}: A_{n, k}^{0} \mapsto H_{n, m}^{k}$ является изометрией c точностью до константы. А именно, при $f_{0} \in A_{n, k}^{0}$

$$
\left\|\psi_{n}^{m-k} f_{0}\right\|^{2}=C_{n-2 k}^{m-k}\left\|f_{0}\right\|^{2} .
$$

Д о к а з а т е л ь с т в о. Искомая формула получается из леммы 6 и следствия 1 прямыми вычислениями. 
В частности, из формул (5), (7) и (12) следует, что коэффициенты $c_{u}$ в (8) задаются формулой $c_{u}^{-2}=C_{n-2 k}^{m-k} \prod_{j=1}^{k}\left(p_{j}-2 j+1\right)\left(p_{j}-2 j+2\right)$.

П р и м е р 3. Пусть $\xi=0101 \ldots$ Тогда $m(n)=[n / 2]$ при любом $n$, и формулы для переходных вероятностей спектральной меры $\mu_{\xi}$ принимают следуюший вид:

(a) если $n$ нечетно, то

$$
\begin{aligned}
\operatorname{Prob}\left(\lambda_{n, k}, \lambda_{n+1, k}\right) & =\frac{n-2 k+2}{2(n-2 k+1)}, \\
\operatorname{Prob}\left(\lambda_{n, k}, \lambda_{n+1, k+1}\right) & =\frac{n-2 k}{2(n-2 k+1)} ;
\end{aligned}
$$

(b) если $n$ четно, то

$$
\operatorname{Prob}\left(\lambda_{n, k}, \lambda_{n+1, k}\right)=\operatorname{Prob}\left(\lambda_{n, k}, \lambda_{n+1, k+1}\right)=\frac{1}{2} .
$$

Эти формулы можно получить и другим способом. Поскольку $\mu_{\xi}$ марковская мера, достаточно найти переходные вероятности для одной таблицы $u$ каждой формы $\lambda=\lambda_{n-k, k}$. Удобно взять в качестве $u$ хорошую таблицу формы $\lambda$ (см. примеры 1 и 2). Заметим, что таблица $v$, полученная из хорошей таблицы $u$ добавлением элемента $n+1$ в первую строку, также хорошая. В нашем случае $\xi$ есть моном $x_{2} x_{4} \cdots x_{2 n}$, и мы видим, что в правую часть формулы (9) этот моном входит ровно один раз, так что $\left(\xi, h_{u}\right)=c_{u}$. Поэтому

$$
\operatorname{Prob}\left(\lambda_{n, k}, \lambda_{n+1, k}\right)=\frac{\left(\xi, h_{v}\right)^{2}}{\left(\xi, h_{u}\right)^{2}}=\frac{C_{n-2 k}^{m(n)-k}}{C_{n+1-2 k}^{m(n+1)-k}},
$$

откуда, с учетом того, что $m(n)=[n / 2]$, следует искомая формула для $\operatorname{Prob}\left(\lambda_{n, k}, \lambda_{n+1, k}\right)$. Очевидно, $\operatorname{Prob}\left(\lambda_{n, k}, \lambda_{n+1, k+1}\right)=1-\operatorname{Prob}\left(\lambda_{n, k}, \lambda_{n+1, k}\right)$.

Интересно сравнить формулы (13), (14) с переходными вероятностями эргодической чентральной меры $\mu_{1 / 2,1 / 2}$ на $T$, соответствующей параметрам Тома $\alpha=\left(\frac{1}{2}, \frac{1}{2}, 0, \ldots\right), \beta=0$ (см., например, [9]).

Лемма 7. Переходные вероятности иентральной меры $\mu_{1 / 2,1 / 2}$ задаются формулой (13) для всех $n$.

Д о к а з а т ель с т в о. Пусть $u \in T_{\lambda}, \lambda \in \mathbb{Y}_{n}$. Тогда

$$
\mu_{1 / 2,1 / 2}\left(C_{u}\right)=s_{\lambda}\left(\frac{1}{2}, \frac{1}{2}\right)=\frac{1}{2^{n}} s_{\lambda}(1,1)=\prod_{\square \in \lambda} \frac{2+c(\square)}{h(\square)},
$$

где $s_{\lambda}$ - функция Шура, $c(\square)$ и $h(\square)-$ содержание и длина крюка клетки $\square \in \lambda$ соответственно и мы воспользовались известной формулой для $s_{\lambda}(1, \ldots, 1)$ (см. [3, пример I.3.4]). Утверждение леммы выводится отсюда при помоши элементарных вычислений. 
Удобно переписать формулы (13), (14), введя замену индексов $j=$ $n-2 k$. В этих обозначениях таблица Юнга задается последовательностью $\left(j_{1}, j_{2}, \ldots\right)$, где $j_{n}$ принимает значения $0,1, \ldots, n$, и переходные вероятности меры $\mu_{\xi}$ равны

$$
\operatorname{Prob}(j, j+1)=\frac{j+2}{2(j+1)}, \quad \operatorname{Prob}(j, j-1)=\frac{j}{2(j+1)}
$$

в нечетный момент времени и

$$
\operatorname{Prob}(j, j+1)=\operatorname{Prob}(j, j-1)=\frac{1}{2}
$$

в четный момент времени. Мы видим, что случайная таблица Юнга с распределением $\mu_{\xi}$ есть траектория неоднородного (и по времени, и по пространству) случайного блуждания на $\mathbf{Z}_{+}$. Таким образом, рассмотренные в данной работе индуцированные представления бесконечной симметрической группы действуют в пространстве функций от траекторий естественньх случайных блужданий. Явные формулы для описания этого действия даются ортогональной формой Юнга (см., например, [2]).

\section{СПИСОК ЛИТЕРАТУРЫ}

1. Джеймс $Г$., Теория представлений симметрических групп. М.: Мир, 1982, 214 с.

2. James G., Kerber A. The Representation Theory of the Symmetric Group. Reading, Mass.: Addison-Wesley, 1981, 510 p.

3. Макдональд И. Симметрические функции и многочлены Холла. М.: Мир, 1985, $222 \mathrm{c}$.

4. Никитин П. П. Реализация неприводимых двустрочечных представлений $S_{n}$ в бесквадратных симметрических формах. - Зап. научн. семин. ПОМИ, 2003, т. 301, c. $212-218$.

5. Tsilevich N.V., Vershik A.M. On different models of representations of the infinite symmetric group. - Adv. Appl. Math., 2006 (to appear).

6. Вериик A. M. Устройство ручных разбиений. - Успехи матем. наук, 1972, т. 27, № 3, c. 195-196.

7. Vershik A.M. Randomization of algebra and algebraization of probability - an attempt at prediction. - Mathematics Unlimited - 2001 and Beyond. Part II. Berlin: Springer-Verlag, 2001, p. 1157-1166.

8. Вершик A.М., Керов С. В. Локально полупростые алгебры. Комбинаторная те-

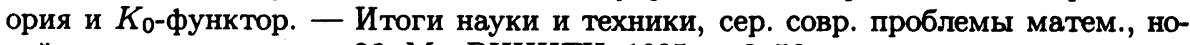
вейшие достижения, т. 26. М.: ВИНИТИ, 1985, с. 3-56.

9. Vershik A.M., Kerov $S . V$. The Grothendieck group of infinite symmetric group and symmetric functions (with the elements of the theory of $K_{0}$-functor of AFalgebras). - Representation of Lie Groups and Related Topics. Ed. by A. M. Vershik and D. P. Zhelobenko. New York: Gordon and Breach, 1990, p. 39-117. (Adv. Stud. Contemp. Math., v. 7.)

10. Вершик A.М., Окуньков А. Ю. Новый подход к теории представлений симметрических групा. II. - Зап. научн. семин. ПОМИ, 2004, т. 307, с. 57-98. 